\title{
Facile and Green fabrication of Multiple Magnetite Nano-cores @Void@Porous Shell Microspheres for Delivery Vehicles
}

\author{
Lei Bi, Gang Pan* \\ Received (in $X X X, X X X)$ Xth $X X X X X X X X X 20 X X$, Accepted Xth $X X X X X X X X X 20 X X$ \\ ${ }_{5}$ DOI: 10.1039/b000000x
}

Multiple magnetite nano-cores@void@porous shell microspheres have been fabricated by using the algae cell wall as the hollow porous shell precursor and the intracellular substances as the reducing agents to form magnetite cores 10 inside the microspheres via hydrothermal reactions. This method not only simplified the fabricating steps and superseded harmful chemical reagents, but also endow the microspheres with a uniform size $(\sim 2.5 \mathrm{um})$, porous shell $(\sim 15$ nm), multiple magnetite nano-cores $(\sim 25 \mathrm{~nm})$ and high void 15 volume ratio $(>\mathbf{7 0} \%)$. The product presents fast magnetic separation and redispersibility as well as $\mathrm{pH}$-switched protein auto-loading (high capacity $>600 \mathrm{mg} \mathrm{g}^{-1}$ ) and unloading as high performance deliver vehicles.

The synthesis of the magnetic core@void@shell rattle type ${ }^{1}$ (or 20 so called yolk-shell ${ }^{2}$ ) micro/nano spheres (MCRSs) have stimulated great interest not only for their appealing structures but also for many technological applications, including: microwave adsorption, catalysis, magnetically-triggered drug release and so on. ${ }^{3}$ These promising applications profit from the 25 novel structure of a magnetically responsive core, functional shell ${ }^{4}$ and void space for loading guest materials ${ }^{5}$. Recently, many attentions have been received on the fabrication of MCRSs using selective etching or dissolution, soft template, Ostwald ripening and ship in bottle methods. ${ }^{6}$ These methods are primarily ${ }_{30}$ based on the pre-core/post-shell or pre-shell/post-shell strategy. ${ }^{7}$

In spite of the success of these works in fabricating MCRSs, there are still some difficult problems that need to be solved. For instance, 1) most of these MCRSs have only one big magnetic core, which left limited space in the cavity. Actually, multiple 35 small cores in sufficient void space (high volume ratio) are more advantageous than one big core in limited void space ${ }^{8}$, because multiple small cores can improve the physical and chemical interactions between the particles and the target objects by adding more reactive sites, furthermore, sufficient void space can be

40 used to load more guest materials. However, it is still a challenge to prepare rattle type microspheres with multiple magnetic cores and high void volume ratio. 2) in order to fabricate complex core@void@shell configuration, tedious procedures, harsh reaction conditions, and environmental harmful chemicals are 45 often inevitable, which not only limited the advanced function of the material but also make the practical production and application of these materials problematic. These limitations can hardly be lifted without the breakthrough in the existing precore/post-shell and pre-shell/post-shell strategies.

50 Chlorella pyrenoidosa, as an inexpensive, abundant, and renewable native biomaterial resource, has been used to produce biofuel $^{9}$ and named as a green healthy food by $\mathrm{FAO}^{10}$. The cell wall of Chlorella pyrenoidosa is constructed primarily of $\alpha$ cellulose and hemicellulose, which provide physical protection ${ }_{55}$ for the whole cell system ${ }^{11}$. The direct use of the cell wall as the shell precursor may effectively simplify the synthetic step of rattle type microspheres. Some biological extracts have been used as natural reagents for fabrication of magnetic nanoparticles ${ }^{12}$. However, these biological extracts require toxic chemical solvent 60 and complicated extraction processes, direct use of native biomaterial may circumvents these problems ${ }^{13}$. Therefore, we conjecture that the rich intracellular substances of the Chlorella pyrenoidosa may supersede harmful chemical reagents for the formation of the internal nanocores.

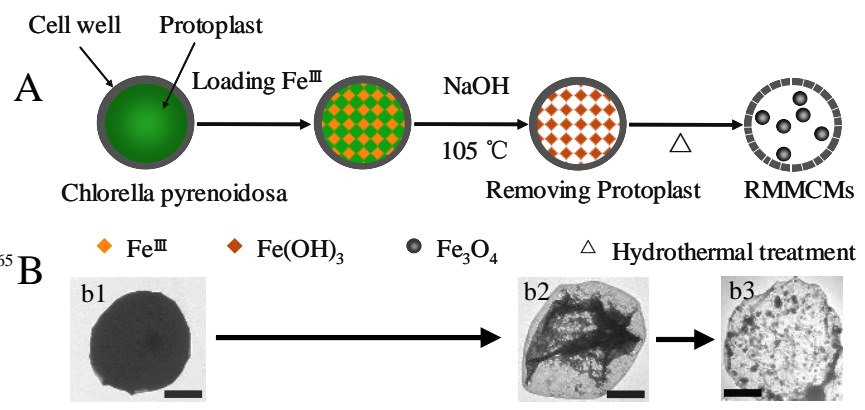

Scheme 1. (A) The fabrication processes for the RMMCMs. (B) The TEM images of the RMMCMs in each step: (b1) Chlorella pyrenoidosa cell, (b2) the cell wall of Chlorella pyrenoidosa with iron hydroxide, (b3) the RMMCMs 70 obtained by the hydrothermal treatment at $200^{\circ} \mathrm{C}$ for $4 \mathrm{~h}$. All scale bars are 1 um.

In this work, we propose a novel method for fabricating rattletype multiple magnetite cores microspheres with porous biopolymer shell (RMMCMs) through mild hydrothermal method $75\left(200^{\circ} \mathrm{C}\right)$. Only three cheap raw materials, i.e. environmentally friendly Chlorella pyrenoidosa cells, ammonium iron citrate, and sodium hydroxide, were used to synthesize the RMMCMs products. The preparing procedure of RMMCMs is illustrated in Scheme 1. Firstly, iron precursor $\left(\mathrm{Fe}^{\mathrm{III}}\right)$ was loaded by dispersing 80 the Chlorella pyrenoidosa powder in the solution of ferric ammonium citrate for $12 \mathrm{~h}$ and then washed off the excess of the iron precursor solution with pure water. Next, the protoplast 
inside the Chlorella pyrenoidosa cells was removed by hot $\left(105^{\circ} \mathrm{C}\right) \mathrm{NaOH}$ solution $(6 \%)$ to get the hollow shell, meanwhile the iron hydroxide of low solubility was formed inside the hollow shell. Finally, the magnetite nano-cores and the porous shell were 5 formed simultaneously by the hydrothermal treatment at $200^{\circ} \mathrm{C}$ for $4 \mathrm{~h}$. The formation process of the RMMCMs could be observed from the TEM images in Scheme 1 (B). The morphology change from spherical Chlorella pyrenoidosa cell (Scheme 1 B (b1)) to hollow shell (Scheme 1 B (b2)) may be due 10 to the fact that protoplast inside the Chlorella pyrenoidosa could be dissolved in hot $\mathrm{NaOH}$ solution but not the cell wall ${ }^{11}$. After hydrothermal treatment, the final product of RMMCMs (Scheme 1B(b3)) was obtained.
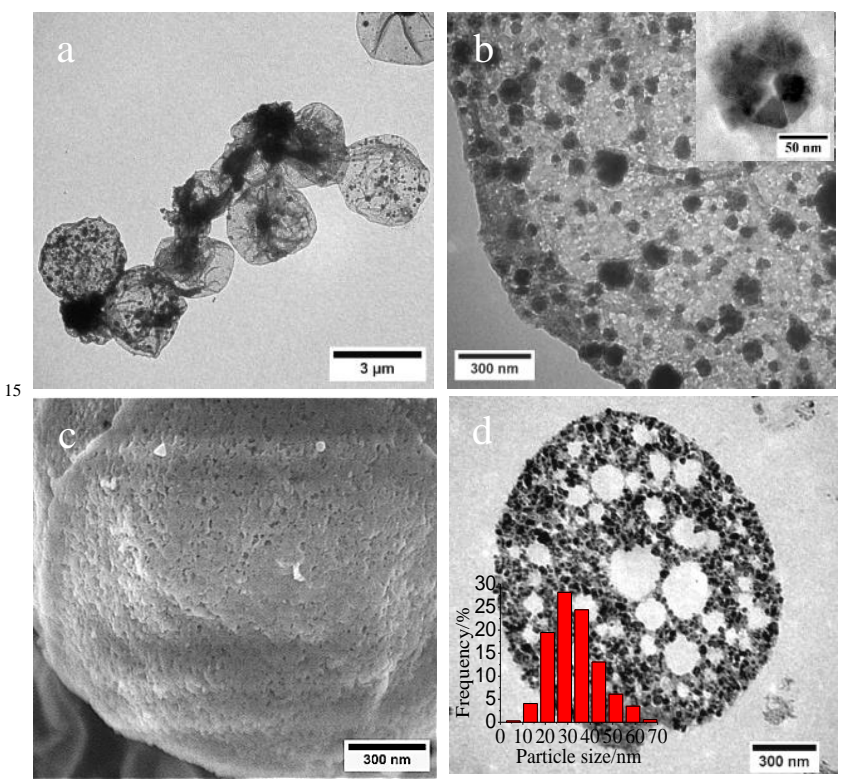

Figure 1. a) and b) TEM images of RMMCMs; the inset in b) is a FETEM image of the particles inside the RMMCMs; c) FESEM image of RMMCMs; d) TEM image of ultramicrotomed a single RMMCMs; the inset in d) is the size distribution of the particles inside the RMMCMs.

20 The TEM image in Figure 1a shows that the microspheres preserve as integral hollow spherical ( 2.5 um) embedded with multiple particles $(\sim 100 \mathrm{~nm})$ with void spaces among the particles and the shell. The void volume ratio of the particles and the void space in the microspheres is about 1:3. The shell thickness is 25 about $40 \mathrm{~nm}$ (Figure 1a) and there are some pores in the shells (Figure 1b). Field emission scanning electron microscopy (FESEM) image further confirms the existence of the pores in the shell (Figure 1c). The $\mathrm{N}_{2}$ adsorption-desorption isotherm of RMMCMs displayed a hysteresis loop, which is characteristics of 30 mesoporous material. Data calculated using the BJH (BarrettJoyner-Halenda) method reveals that the average size of the shell pores was about $15 \mathrm{~nm}$ (Figure S1, Supporting Information). The BET surface area of RMMCMs was $64.1 \mathrm{~m}^{2} \mathrm{~g}^{-1}$. The formation of pores may be due to the differences in the hydrolysis resistance 35 of different components in the cell wall ${ }^{14}$. FETEM (inset in Figure 1b) examination of the particles suggests that these micro particles were made up of several nanoparticles. In order to obtain the actual distribution of the particles and the structures inside the RMMCMs, the microspheres were embedded with 40 epoxy resins and ultramicrotomed into thin slices and analyzed with TEM. Most of the nanoparticles are in the range $20 \mathrm{~nm}$ to 40 nm (inset in Figure 1d) and localized inside the microspheres where many cavities are also observed. In comparison, only few nanoparticles were found on the exterior surface of the 45 microspheres (Figure 1c). Since the particle size is larger than the apertures of the shell, these nanoparticles and their clusters can be well confined inside the microspheres.
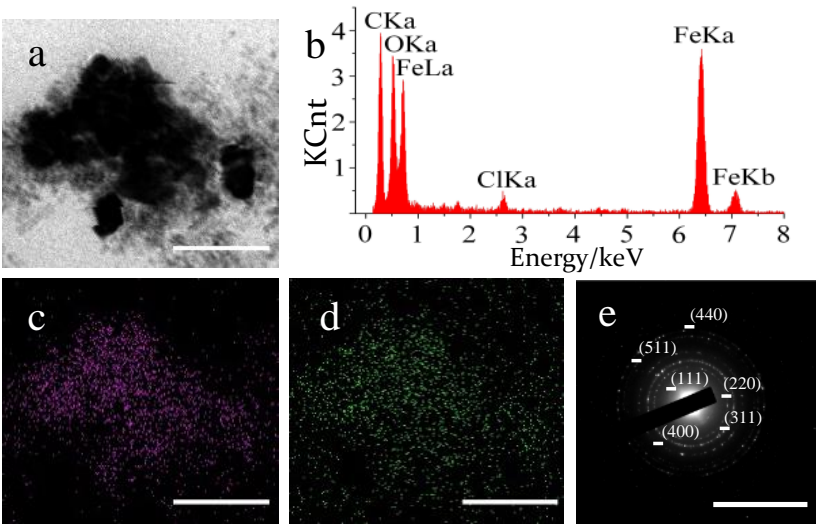

$50 \quad$ Figure 2. a), b), c) and d) FETEM image, EDX spectrum, Elemental maps of $\mathrm{Fe}$ and $\mathrm{O}$ and SAED pattern of the nanoparticles inside the RMMCMs, respectively. All scale bars are $100 \mathrm{~nm}$.

In order to identify the structure and composition of the nanoparticles inside the RMMCMs, energy-dispersive X-ray 55 (EDX) spectrum, elemental mapping characterization and selected-area electron diffraction (SAED) were carried out by using JEM 2100F field emission transmission electron microscope. Figure 2a displays the normal FETEM image of the nanoparticles inside the RMMCMs. Peaks of $\mathrm{C}, \mathrm{O}, \mathrm{Fe}$ and $\mathrm{Cl}$ are ${ }_{60}$ identified from the EDX spectrum (Figure $2 b$ ). The $\mathrm{C}$ and $\mathrm{Cl}$ peaks can be attributed to epoxy resin and hydrochloric acid that are used in the sample treatment. The $\mathrm{Fe}$ and $\mathrm{O}$ peaks can only be assigned to the nanoparticles (Figure 2c, 2d). In Figure 2e, the SAED pattern contains at least six well-defined spotted rings, the 65 spotted appearance of the diffraction rings is due to high crystallinity of the obtained nanoparticles ${ }^{15}$. The corresponding lattice spacing calculated from the SAED pattern (Table S1, Supporting Information) are consistent with the standard magnetite (JCPDS no. 99-0073).
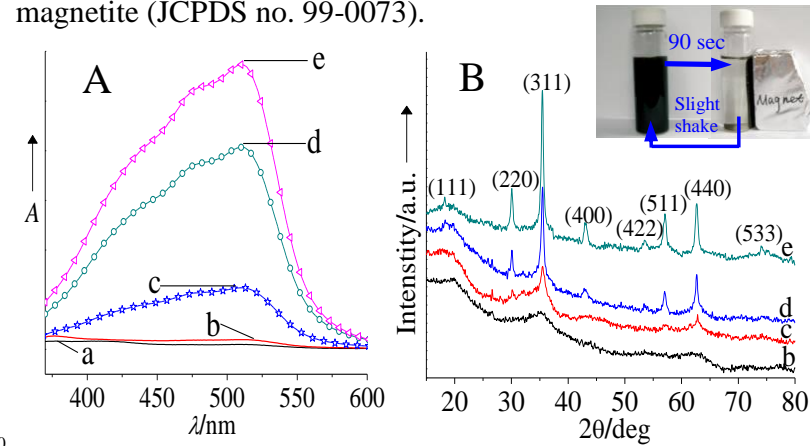

Figure 3. UV/Vis spectra (A) of $\mathrm{Fe}^{\amalg}$-phen acid solutions obtained by adding 1, 10-phenanthroline in the acid extract of the powder samples (a-e). XRD patterns (B) of the powder samples (b-e). (a) Chlorella pyrenoidosa containing $\mathrm{Fe}^{\mathrm{III}}$; (b) obtained by removing the protoplast of sample (a) under hot $\left(105^{\circ} \mathrm{C}\right)$ $75 \mathrm{NaOH}$ solution $(6 \%)$; (c), (d) and (e) were prepared by hydrothermal treatment of sample (b) under $170^{\circ} \mathrm{C}, 185^{\circ} \mathrm{C}$ and $200^{\circ} \mathrm{C}$, respectively. The inset pictures in (B) show that the RMMCMs $\left(200^{\circ} \mathrm{C}\right)$ possess fine magnetic responsivity and redispersibility. 
During the synthesis procedures of RMMCMs, $\mathrm{Fe}^{\text {III }}$ was used as the single iron precursor, but the magnetite contains both $\mathrm{Fe}^{\mathrm{II}}$ and $\mathrm{Fe}^{\mathrm{III}} \cdot{ }^{16}$ It is necessary to confirm whether $\mathrm{Fe}^{\mathrm{II}}$ exist in the RMMCMs products. $\mathrm{Fe}^{\mathrm{II}}$ salts in acidic solutions react with 1, 105 phenanthroline to form a dark red complex (Fe ${ }^{\mathrm{II}}$-phen), and the maximum absorption wave length of this complex is $510 \mathrm{~nm}$. Fe salts do not react under these conditions ${ }^{16}$. The UV-vis spectra were used to identify the existence of the $\mathrm{Fe}^{\mathrm{II}}$-phen compound. $\mathrm{XRD}$ was used to research the phase transition of RMMCMs 10 under different reaction temperatures. Figure $3 \mathrm{~A}(\mathrm{a})$ shows that, after loading Chlorella pyrenoidosa with $\mathrm{Fe}^{\mathrm{II}}$, no $\mathrm{Fe}^{\mathrm{II}}$-phen complex was detected, which means that $\mathrm{Fe}^{\mathrm{III}}$ had not been reduced in this stage. After the protoplast was removed by hot $\mathrm{NaOH}$ solution, only a tiny amount of $\mathrm{Fe}^{\mathrm{II}}$-phen complex 15 appeared (Figure 3A(b)). The XRD patterns revealed that no distinct diffraction peaks were observed (Figure 3B(b)). According to Majzlan and co-workers ${ }^{17}$, the amorphous ferrihydrite is the main form of iron under such condition. When the temperature was raised to $170^{\circ} \mathrm{C}$, a certain amount of $\mathrm{Fe}^{\mathrm{II}}$ 20 phen complex were found (Figure $3 \mathrm{~A}(\mathrm{c})$ ). And two main characteristic peaks of magnetite at the position of 35.4 (311) and 65.6 (440) appeared (Figure 3B(c)). The broad diffraction peaks suggests that the crystallinity of the sample is poor. The amount of $\mathrm{Fe}^{\mathrm{II}}$-phen complex significantly increased and several new 25 diffraction peaks were present and all the peaks matched well with magnetite (Table S1, Supporting Information) with the increase of temperature from 185 to $200^{\circ} \mathrm{C}$ (Figure $3 \mathrm{~A}(\mathrm{~d})$ and $3 \mathrm{~A}(\mathrm{e}))$. The results are consistent with existing reports on the effect of $\mathrm{Fe}^{\mathrm{II}} / \mathrm{Fe}^{\mathrm{III}}$ molar ratio on the formation of magnetite ${ }^{18}$.

30 However, without the adding of Chlorella pyrenoidosa, the final products were pure hematite (Figure S3, Supporting Information), when all the other experimental conditions were kept the same. Therefore, the addition of Chlorella pyrenoidosa is the key factor for the green fabrication of magnetite nanoparticles. It is known 35 that many substances in the native biomaterials have the reduction ability ${ }^{19}$, but further studies are needed to identify which substances are responsible for the production of magnetite nanoparticles. The inset pictures in Figure 3B) show that the black RMMCMs $\left(200{ }^{\circ} \mathrm{C}\right)$ were separated from the solution by 40 the magnet within $90 \mathrm{~s}$ and re-dispersed quickly with a slight shake once the magnetic field was removed.
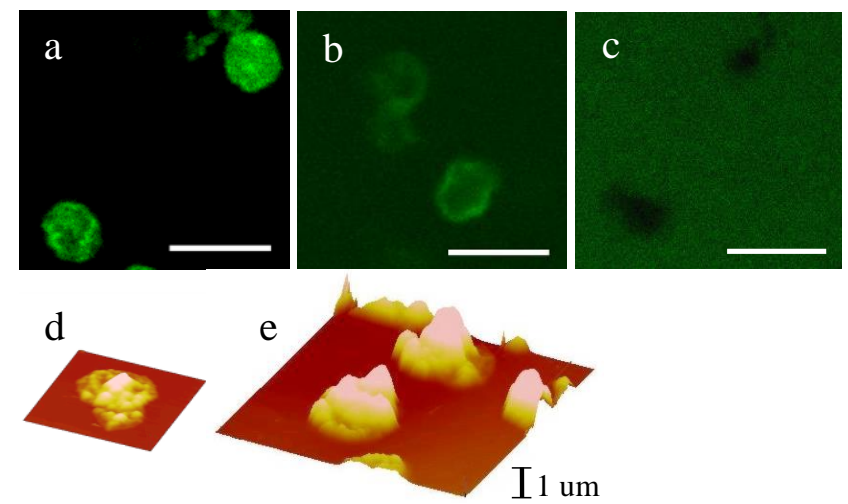

Figure 4. CLSM images of a) auto-loading of FITC-BSA inside the 45 RMMCMs at $\mathrm{pH} \mathrm{5;} \mathrm{b)} \mathrm{and} \mathrm{c)} \mathrm{release} \mathrm{of} \mathrm{the} \mathrm{encapsulated} \mathrm{FITC-BSA} \mathrm{from}$ RMMCMs at $\mathrm{pH} 7$ and $\mathrm{pH} 9$, respectively; d) $(5 \times 5 \mathrm{um})$ and e) $(10 \times 10 \mathrm{um}) \mathrm{SFM}$ images before and after loading of BSA into the RMMCMs at $\mathrm{pH} 5$. All scale bars are 5 um.
In order to test the potential application of the RMMCMs, 50 bovine serum albumin (BSA) was chosen as a model protein to test its delivery vehicle effect. Confocal laser scanning microscopy (CLSM) was used to obtain the detailed and visible information of the encapsulation and release properties of FITCBSA (fluorescein isothiocyanate labelled BSA) as a function of ${ }_{55} \mathrm{pH}$. At $\mathrm{pH} 5$, after adequate mixing of FITC-BSA and RMMCMs, the fluorescence intensity inside the RMMCMs was much higher than that in the bulk solution (Figure 4a). The scanning force microscopy (SFM) images display the significant difference in the volume of RMMCMs before and after BSA encapsulation ${ }_{60}$ (Figure $4 \mathrm{~d}$ and $4 \mathrm{e}$ ), which demonstrated that BSA could be auto loaded into the RMMCMs at $\mathrm{pH} 5$ and the protein loading capacity reached to $>600 \mathrm{mg} \mathrm{g}^{-1}$. After the RMMCMs with FITC-BSA were re-dispersed in $\mathrm{pH} 7$ buffer solution, part of FITC-BSA was released from the RMMCMs and diffused into 65 the surrounding solution (Figure $4 \mathrm{~b}$ ). When the $\mathrm{pH}$ value was further increased to 9, the interior of the RMMCMs was dark and the fluorescence intensity of the bulk medium was much higher (Figure 4c), which suggests that most of the FITC-BSA is out of the RMMCMs. This can be explained by the electrostatic forces 70 interaction among the FITC-BSA and the multiple magnetite cores within the RMMCMs at different $\mathrm{pH}$ values (Figure S4, Supporting Information).

In summary, a novel method was developed to prepare rattle type microspheres with multiple magnetite nano-cores and porous 75 shell. By controlling the hydrothermal synthetic conditions, the cell wall of the Chlorella pyrenoidosa formed the porous biopolymer shell, and the iron precursor $\left(\mathrm{Fe}^{\mathrm{III}}\right)$ was partly reduced and turned into magnetite nanoparticles inside the porous shell with the assistance of Chlorella pyrenoidosa. The multiple 80 magnetite cores not only endow the microspheres with excellent magnetic responsivity, but also play a significant role on the auto loading or release of protein into/out of the RMMCMs under different $\mathrm{pH}$ conditions. The method advanced the conventional synthesis methods, which make it possible to supersede harmful 85 chemical reagent with algae protoplast. The RMMCMs may have potential applications including encapsulation of active ingredients, magnetic separation of biological molecules and magnetic drug target delivery.

\section{Acknowledgements}

90 The study was supported by Chinese National Basic Research Program (2010CB933600) and the Science Promotion Program of RCEES, CAS (YSW2013B05). We thank Dr. L. Wang for her help in HRTEM measurement

\section{Notes and references}

95 Department of Environmental Nanomaterials, Research Center for Ecoenvironmental Sciences, Chinese Academy of Sciences, Beijing 100085, (P. R. China). Fax: (+86)10-6292-3541; Tel: (+86)10-6284-9686; E-mail: gpan@rcees.ac.cn (G.P.)

$\uparrow$ Electronic Supplementary Information (ESI) available: Experimental 100 details and relevant figures. See DOI: 10.1039/b000000x/

1 G. L. Li, H. Mohwald, D. Shchukin, Chem. Soc. Rev, 2013, 42, 3628.

2 J. Liu, S. Z. Qiao, J. S. Chen, X. W. Lou, X. G. Xing, G. Q. Lu, Chem. Commun, 2011, 47, 12578.

3 (a) J. Liu, J. R. Cheng, C. Xu, J.M. Liu, Z. Liu, Appl. Mater. Interfaces, 105 2013, 5, 2503. (b) Y. Ye, L. Kuai, B. J. Geng, Mater. Chem, 2012, 22, 
19132. (c) S. H. Hu, Y. Y. Chen, T. C. Liu, T. Tung, H. Liu, D. M. S. Y. Chen, Chem. Commun, 2011, 47, 1776.

4 D. Shchukin, H. Mohwald, Science, 2013, 341, 3458.

5 J. Liu, S. Z. Qiao, Q. H. Hu, G. Q. Lu, Small, 2011, 7, 425.

56 (a) X. Zhang, L. J. Jiang, Mater. Chem, 2011, 21, 10653; (b) Q. Fang, S. Xuan, W. Jiang, X. Gong, Adv. Funct. Mater, 2011, 21, 1902; (c) J. Liu, S. Z. Qiao, S. B. Hartono, G. Q. Lu, Angew. Chem. Int. Ed, 2010, 49, 4981; (d) L. Zhang, S. Z. Qiao, Y. G. Jin, Z. Chen, H. C. Gu, G. Q. Lu, Adv. Mater, 2008, 20, 805; (e) L. Guo, J. Li, L. Zhang, J. Li, 10 Y. Li, C. Yu, J. Shi, M. Ruan, J. J. Feng, Mater. Chem, 2008, 18, 2733.

7 L. Qiang, X. Meng, L. Li, D. Chen, X. Ren, H. Liu, J. Ren, C. Fu, T. Liu, F. Gao, Y. Zhang, F. Tang, Chem. Commun, 2013, 49, 7902.

8 W. M. Zhang, J. S. Hu, Y. G. Guo, S. F. Zheng, L. S. Zhong, W. G.

15 Song, L. J. Wan, Adv Mater, 2008, 20, 1160.

9 D. R. Georgianna, S. P. Mayfield, Nature. 2012, 488, 329.

10 Y. Shi, J. Sheng, F. Yang, Q. Hu, Food Chem, 2007, 103, 101.

11 D. H. Norchtort, K. J. Goulding, J. Biochem, 1958, 70, 391.

12 (a) S. Gao, Y. Shi, S. Zhang, K. Jiang, S. Yang, Z. D. Li, E. T.

20 Muromachi, J. Phys. Chem. C, 2008, 112, 10398; (b) J. R. Chiou, B. H. Lai, K. C. Hsu, D. H. Chen, J. Hazard. Mater, 2013, 248, 394.

13 (a) Z. Schnepp, Angew Chem. Int. Ed, 2013, 52, 1096; (b) Z. Schnepp, W. Yang, M. Antonietti, C. Giordano, Angew. Chem, Int. Ed. 2010, 49, 6564.

2514 D. Z. Ni, L. Wang, Y. H. Sun, Z. R. Guan, S. Yang, K. B. Zhou, Angew. Chem. Int. Ed, 2010, 49, 4223.

15 D. Caruntu, G. Caruntu, Y. Chen, C. J. O'Connor, G. Goloverda, V. L. Kolesnichenko, Chem. Mater, 2004, 16, 5527.

16 S. Laurent, D. Forge, M. Port, A. Roch, C. Robic, L. Vander Elst, R. N. Muller, Chem. Rev, 2008, 108, 2064.

17 J. Majzlan, A. Navrotsky, U. Schwertmann, Geochim. Cosmochim. Acta, 2004, 68, 1049.

18 N. Mizutani, T. Iwasaki, S. Watano, T. Yanagida, H. Tanaka, T. Kawai, Bull. Mater. Sci, 2008, 31, 713.

3519 R. Shukla, S. K. Nune, N. Chanda, K. Katti, S. Mekapothula, R. R. Kulkarni, W. V. Welshons, R. Kannan, K. V. Katti, Small, 2008, 4, 1425 .

Microspheres with multiple magnetite cores, porous ${ }_{40}$ shell and high volume fabricated from hydrothermal treated algae cells and single iron source $\left(\mathrm{Fe}^{\mathrm{II}}\right)$.

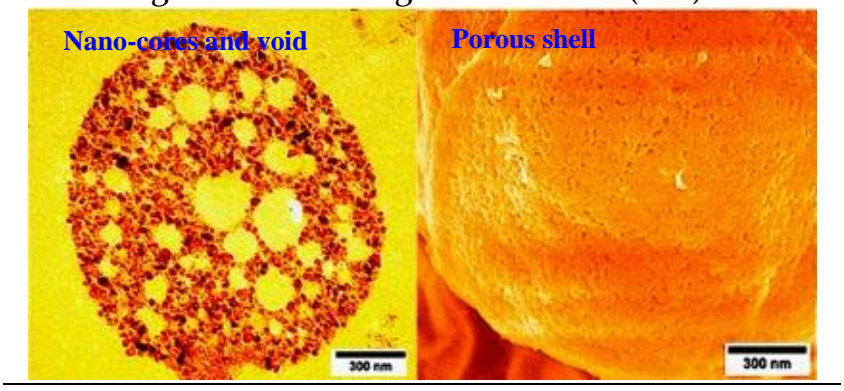

